\title{
VITAE: \\ RECUPERAÇÃO DE OBJETOS DE APRENDIZAGEM \\ BASEADA NA WEB 2.0
}

\author{
VITAE: \\ LEARNING OBJECTS RETRIEVAL BASED ON WEB 2.0
}

\begin{abstract}
Resumo
Objetos de aprendizagem (OA) são materiais digitais utilizados para apoiar o ensino e a aprendizagem. Tradicionalmente, OAs são desenvolvidos como material de apoio a professores. Entretanto, eles podem ser utilizados por alunos ou qualquer pessoa interessada em aprender um determinado assunto. OAs podem ser encontrados em repositórios ou na Web, em sites de docentes e de instituições de ensino. Apesar disso, nem sempre os OAs são facilmente encontrados. Em geral, os repositórios abordam poucos campos do conhecimento e poucas opções de línguas. As máquinas de busca retornam um grande volume de informação, o que dificulta, para o usuário, identificar os OAs que são relevantes para as suas necessidades. Nesse contexto, este artigo propõe um mecanismo para recuperação de OAs, baseado nos princípios da Web 2.0. Dentre as opções de aplicações Web 2.0, o modelo de bookmarking social se destaca por facilitar o armazenamento de links a OAs e por permitir o compartilhamento e a classificação desses links. Além disso, ao contrário do que ocorre com os repositórios, essas ferramentas permitem que qualquer usuário possa postar novos links, sem restrições. O protótipo dessa ferramenta foi denominado VITAE. VITAE oferece o serviço de bookmarking social, de forma que usuários possam armazenar, organizar, avaliar e compartilhar links com outras pessoas.
\end{abstract}

Palavras-chave: Tecnologia educacional. Informática e educação. Internet.

\begin{abstract}
Digital materials used in teaching and learning are called learning objects (LO). Traditionally, LOs were developed for use as support materials for teacher. However, they can be used by students or whoever is interested in learning something. LOs can be found in repositories and also on the Web, in teachers and institutions sites. In spite of that, they are not easily found. Repositories address few knowledge fields and few languages. Search engines return a large amount of information that makes it difficult for users to identify relevant LOs to meet their goals. In this context, this paper proposes a new mechanism for LOs retrieval, based on Web 2.0 principles. Among the options for Web 2.0

\footnotetext{
1 Mestre em Informática pela PUC-Minas e professora do Instituto Federal de Minas Gerais. E-mail: geovalia.santos@ifmg.edu.br - São João Evangelista, MG, Brasil.

${ }^{2}$ Doutora em Ciências da Computação pela UFMG e professora do Mestrado em Informática da Pontifícia Universidade Católica de Minas Gerais (PUC Minas). E-mail: lucila@pucminas.br - Belo Horizonte, MG, Brasil.

${ }^{3} \mathrm{PhD}$ em Ciência da Computação pela Universidade de Waterloo, Canadá. Professora do Instituto de Ciências Exatas e Informática da Pontifícia Universidade Católica de Minas Gerais (PUC Minas). E-mail: guta@pucminas.br - Belo Horizonte, MG, Brasil.

Recebido em: 14/09/2011 / Aprovado em: 22/05/2012.
} 
applications, the model of social bookmarking called our attention because it provides facilities to store links to LOs, and also to share and classify these links. Moreover, unlike what happens with the repositories, in these tools, any user can post new links, without restrictions. The prototype of this tool was called VITAE. VITAE provides links to LOs, in a way that people can store, organize and share them with others.

Keywords: Computer uses in education. Educational technology. Internet.

\section{INTRODUÇÃO}

O Institute of Electrical and Electronics Engineers (IEEE) definiu objeto de aprendizagem (OA) como "qualquer entidade, digital ou não-digital, que pode ser utilizada para aprendizagem, educação ou treinamento" (LEARNING TECHNOLOGY STANDARDS COMMITTEE, 2002, p. 6). Como essa definição é muito ampla, neste trabalho, os objetos de aprendizagem estarão restritos aos materiais digitais utilizados na educação.

Um OA pode auxiliar professores em suas atividades e também alunos no processo de construção de seu conhecimento. Entretanto, o desenvolvimento de um OA é uma tarefa difícil ou trabalhosa para os professores (FABRE; TAROUCO; TAMUSIUNAS, 2003; GONZALEZ-BARBONE; ANIDO-RIFON, 2008), pois, em geral, estes não possuem os conhecimentos técnicos e pedagógicos necessários para criar OAs que satisfaçam aos critérios de avaliação existentes. Além disso, a falta de tempo e de suporte por parte das instituições de ensino aumenta a dificuldade de produção de OAs.

Considerando esse contexto, uma forma de expandir o uso de OAs é fornecer recursos para pesquisa, recuperação e reúso de objetos já existentes. Atualmente, dois recursos amplamente utilizados são os repositórios e as máquinas de busca.

Com relação aos repositórios de OAs, nem sempre eles são conhecidos ou facilmente encontrados. Quando são conhecidos, são heterogêneos e distribuídos, porque cada um possui suas próprias características, devido à falta de padronização entre eles. Até o momento, não se conhecem propostas de padronização de repositórios, nem mesmo na especificação do Sharable Courseware Object Reference Model (SCORM) (ADVANCED DISTRIBUTED LEARNING, 2004). Um outro aspecto negativo dos repositórios é o número pequeno de OAs desenvolvidos em língua diferente do inglês. No caso específico da língua portuguesa, somase ao pequeno número de objetos o fato de que estes endereçam poucas áreas do conhecimento e poucos segmentos da educação. 
OAs também podem ser encontrados na Web, por meio de máquinas de busca, em sites que não foram desenvolvidos com o objetivo de prestar o serviço de repositório. Como exemplos, podem-se citar páginas pessoais de professores ou sites que oferecem cursos que podem ser baixados ou que são on-line, como os oferecidos pela $\mathrm{BBC}^{4}$. Entretanto, nem sempre é fácil encontrar OAs na Web (COELHO et al., 2008). Um dos motivos é a quantidade de links que uma máquina de busca retorna, quantidade essa que pode ultrapassar um milhão de resultados.

Para minimizar essas dificuldades, este artigo apresenta uma proposta de ferramenta que tem por objetivo apoiar o usuário em sua busca por objetos de aprendizagem relacionados ao que deseja ensinar ou aprender. Essa ferramenta foi denominada VITAE, de Virtual and Interesting Teaching and learning material for Always and Ever, porque ela pode apoiar também a aprendizagem contínua (COELHO et al., 2009). VITAE segue os princípios da Web 2.0, porque esses princípios possuem características positivas para a recuperação de informação. Por exemplo, ferramentas Web 2.0 oferecem resultados melhores do que a pesquisa por OAs, utilizando mecanismos tradicionais (COELHO et al., 2008).

Dentre os possíveis modelos de ferramentas Web 2.0, foi escolhido o de bookmarking social, que permite aos usuários armazenar links para páginas e identificá-los com palavras-chave ou tags, denominadas bookmarks ou favoritos. Ferramentas de bookmarking social são simples e intuitivas, porque reforçam um conceito que existe nos navegadores Web, como o Internet Explorer e o Mozilla Firefox, que é o de armazenamento de links favoritos. Além disso, as ferramentas de bookmarking social permitem o compartilhamento de bookmarks ou links favoritos, apresentando também avaliações dos OAs realizadas pelos seus usuários.

Com esse mecanismo, professores e alunos podem recuperar OAs que se encontram espalhados na Web, tais como apresentações, relatórios técnicos, animações e outros materiais que nem sempre são armazenados em repositórios. Assim, OAs podem ser reutilizados, reduzindo investimentos financeiros e de tempo, necessários para o desenvolvimento de novos OAs.

Este artigo está estruturado da seguinte forma: a Seção 2 apresenta o conceito e as principais características e ferramentas da Web 2.0 que são importantes para este trabalho; a Seção 3 apresenta uma discussão sobre métodos de recuperação de OAs; a Seção 4 apresenta

\footnotetext{
${ }^{4}$ Disponível em: 〈www.bbc.co.uk/languages〉. Acesso em: 20 set. 2010. 
as principais funcionalidades do VITAE; a Seção 5 apresenta os resultados de uma avaliação do VITAE; e, finalmente, a Seção 6 apresenta as conclusões e as sugestões para trabalhos futuros.

\section{WEB 2.0}

Web 2.0 não é uma nova tecnologia, mas uma maneira mais participativa de utilizar a Web e uma nova forma de ver a Web como uma plataforma (O'REILLY, 2005). Alguns exemplos bem conhecidos de aplicativos para a Web 2.0 são: YouTube ${ }^{5}$, Facebook ${ }^{6}$, Delicious $^{7}$, Google Docs ${ }^{8}$ e Wikipedia ${ }^{9}$.

Uma das características da Web 2.0 é o suporte que oferece a serviços de grupo: atividades colaborativas e compartilhamento de dados. A pesquisa por informação não é baseada em metadados, mas em $\operatorname{tags}^{10}$. A uma coleção de tags criadas colaborativamente atribui-se o nome de "folksonomia".

Dentre todos os princípios da Web 2.0, três contribuem para a recuperação de objetos de aprendizagem:

(i) Conteúdo gerado pelo usuário - na Web 2.0, todo usuário pode contribuir para aumentar a quantidade de informação disponível, pela inclusão de materiais diversos na rede, tais como vídeos, animações, imagens, textos, links. De acordo com o site Alexa ${ }^{11}$, que publica diariamente um ranking dos um milhão de sites mais acessados no mundo, uma das ferramentas de bookmarking social mais popular é o Delicious, que armazena mais de 200 novos links por minuto. Um outro site da lista é o YouTube, que informa que, no ano de 2010, foram postados mais de 13 milhões de horas de vídeo, o que representa uma média de 35 horas de vídeo a cada minuto. Cabe ainda destacar o exemplo da Wikipédia, que é escrita colaborativamente por mais de 14 milhões de voluntários e totalizou, em 2010,

\footnotetext{
${ }^{5}$ Disponível em: <www.youtube.com>. Acesso em: 20 set. 2010.

${ }^{6}$ Disponível em: 〈www.facebook.com $>$. Acesso em: 20 set. 2010.

${ }^{7}$ Disponível em: 〈www.delicious.com $>$. Acesso em: 20 set. 2010.

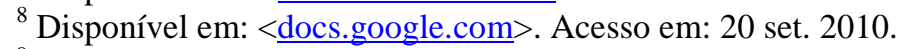

${ }^{9}$ Disponível em: 〈www.wikipedia.org〉. Acesso em: 20 set. 2010.

${ }^{10}$ Tags foram definidas como descritores de texto curtos, escolhidos livremente por usuários.

${ }^{11}$ Disponível em: <www.alexa.com>. Acesso em: 20 set. 2010.
} 
mais de 3 milhões de artigos em inglês. Esses exemplos demonstram o potencial da Web 2.0 para armazenar, organizar e compartilhar conteúdo educacional.

(ii) Melhor aproveitamento da inteligência coletiva - a ideia por trás desse princípio é a de que, em algumas situações, "o povo" tem mais chances de oferecer uma resposta correta, do que um indivíduo (ANDERSON, 2007). Por exemplo, em um site de turismo, se um hotel é classificado como de duas estrelas, mas todos atribuem a ele notas 8 ou 9 e todos os comentários são positivos, podemos concluir que o hotel não é luxuoso, mas provavelmente é bom. Então, no contexto educacional, a possibilidade de atribuir notas a objetos de aprendizagem pode aumentar a confiança de um usuário na qualidade e na relevância do material acessado.

(iii) Valorização da cauda longa - a Web possui diversos sites dispersos, com conteúdos que podem interessar a percentualmente poucos usuários, mas que, em conjunto, representam um grande número de pessoas. Esses sites compõem o que é denominado cauda longa. Por exemplo, se comparado ao inglês, o número de pessoas interessadas em aprender croata ou catalão ou grego é muito pequeno (SAINT IGNATIUS HIGH SCHOOL, 2011). Entretanto, quando somamos o número de pessoas interessadas em aprender uma dessas línguas, o número total obtido é considerável. No contexto educacional, esse princípio amplia as opções de temas a serem ofertados para aprendizagem.

A Web 2.0 oferece diversas ferramentas que podem ser utilizadas para promover a educação. A maior parte delas, como as já citadas Wikipédia e Delicious, não foi desenvolvida especificamente para uso educacional. Por isso, em geral, o material que disponibilizam não satisfazem às características desejáveis para objetos de aprendizagem (NESBIT; BELFER; LEACOCK, 2003). Além disso, a quantidade de material que armazenam dificulta a recuperação e a seleção de material que seja útil para o contexto educacional. Por exemplo, Facebook, a rede social mais utilizada em 2010, possui muitas comunidades que discutem temas educacionais, como os métodos de ensino e aprendizagem, a evolução da educação, a aprendizagem de línguas. Entretanto, ela não oferece nenhum suporte para compartilhamento de OAs. 
Dentre as ferramentas educacionais da Web 2.0, as seguintes merecem destaque, por estarem dentre os sites mais usados, segundo o Alexa Traffic Rank ${ }^{12}$ :

- Edmodo ${ }^{13}$ é um site de micro-blogging para escolas, educadores e alunos. Ela dá suporte para o compartilhamento, entre professores e seus alunos, de material educacional, links, enunciados de trabalhos, notas e notícias. Entretanto, não permite o compartilhamento de material entre professores.

- Edutopia ${ }^{14}$ é uma comunidade de professores e outras pessoas interessadas em mudar a educação. Ela tem por objetivo a discussão e a disseminação de práticas inovadoras. Apesar de sua contribuição para a evolução das práticas educacionais, ela não oferece suporte para compartilhamento de objetos de aprendizagem.

- Elluminate ${ }^{15}$ oferece suporte para conferências via Web e rede social. Uma de suas desvantagens reside no fato de não ser uma ferramenta gratuita.

- $\quad$ Italki $^{16}$ é uma rede social para pessoas que desejam ensinar ou aprender línguas. De acordo com informação disponível em sua página principal, seus membros pertencem a mais de 200 países. Eles se comunicam e compartilham material para aprendizagem de mais de 100 línguas. Italki é uma ferramenta livre, mas oferece suporte para aulas pagas, quando um aluno se conecta a um professor. Todo o material educacional é restrito ao tema de línguas.

- Learnhub ${ }^{17}$ é uma rede de aprendizagem social que tem por objetivo auxiliar pessoas que desejam estudar em outros países. Ela oferece banco de questões de exames internacionais, como o TOEFL. Também fornece informação sobre instituições de ensino, programas internacionais e custo de vida em diversos países. Os estudantes também podem interagir com outros professores e alunos.

- Sites For Teachers ${ }^{18}$ é um site que permite a seus usuários armazenar links para sites educacionais, que são classificados por popularidade. Não há outro tipo de avaliação, como, por exemplo, da qualidade do material educacional disponibilizado. Seu uso é gratuito. O mecanismo de recuperação é muito simples, baseado em palavras-chave.

\footnotetext{
${ }^{12}$ Disponível em: <www.alexa.com>. Acesso em: 21 set. 2010.

${ }^{13}$ Disponível em: 〈www.edmodo.com>. Acesso em: 21 set. 2010.

${ }^{14}$ Disponível em: $\langle\underline{w w w . e d u t o p i a . o r g}\rangle$. Acesso em: 21 set. 2010.

${ }^{15}$ Disponível em: <www.elluminate.com $>$. Acesso em: 21 set. 2010.

${ }^{16}$ Disponível em: 〈www.italki.com>. Acesso em: 21 set. 2010.

${ }^{17}$ Disponível em: <learnhub.com>. Acesso em: 21 set. 2010.

${ }^{18}$ Disponível em: 〈sitesforteachers.com>. Acesso em: 21 set. 2010.
} 
- TeachersNet ${ }^{19}$ oferece um espaço onde as pessoas podem compartilhar artigos e discutir práticas de ensino-aprendizagem. Também permite o compartilhamento de planos de aula, que são organizados por categorias. Um problema reside no fato de que todo o material é organizado em uma lista, o que pode dificultar a recuperação de um plano de aula específico, à medida que a lista vai se tornando mais extensa.

- TeacherTube é uma comunidade on-line que tem por objetivo ajudar professores a compartilhar objetos de aprendizagem. Ela organiza material educacional por tipo: vídeo, documentos, áudio, fotos, blogs e outros recursos. Usuários podem contribuir com comentários. Mas não há outro tipo de filtro ou possibilidade de avaliação que possa ajudar professores a encontrar e selecionar objetos de aprendizagem.

- Wepapers $^{21}$ é uma rede social que permite que alunos compartilhem e discutam artigos, provas, soluções de exercícios e anotações. Entretanto, o material postado não é avaliado. Por isso, os resultados de uma consulta são organizados de acordo, apenas, com a data de postagem do material.

Apesar da diversidade de opções, nenhuma dessas ferramentas permite que os usuários avaliem OAs de acordo com métodos consistentes de avaliação. Além disso, no caso de uma consulta, a maior parte dessas ferramentas retorna uma grande quantidade de resultados, o que dificulta a seus usuários selecionar o OA desejado. Portanto, alunos e professores necessitam de uma ferramenta que atenda melhor à necessidade de pesquisa e recuperação de OAs.

\section{RECUPERAÇÃO DE OBJETOS DE APRENDIZAGEM}

Objetos de aprendizagem (OA) podem ser recuperados de repositórios ou por meio de máquinas de busca.

Repositórios armazenam coleções de OAs. Para que um OA seja incluído em um repositório, ele é avaliado por um comitê responsável pelo repositório. Essa avaliação considera diferentes aspectos do OA, tais como metadados, formato, objetivos e outros. Se o OA for aprovado, o repositório mantém uma cópia do OA para que outros usuários possam

\footnotetext{
${ }^{19}$ Disponível em: <teachers.net>. Acesso em: 21 set. 2010.

${ }^{20}$ Disponível em: $\langle\underline{w w w 1 . t e a c h e r t u b e . c o m}>$. Acesso em: 21 set. 2010.

${ }^{21}$ Disponível em: 〈www.wepapers.com>. Acesso em: 21 set. 2010.
} 
acessá-lo. Os OAs armazenados podem ser recuperados por meio de ferramentas disponibilizadas pelos próprios repositórios. Entretanto, os repositórios nem sempre possuem OAs na língua desejada ou na área específica de interesse. Em repositórios mais conhecidos, como o Multimedia Educational Resources for Learning and Online Teaching (MERLOT) e o National Sciences Digital Library (NSDL), a maior parte dos OAs foi desenvolvido em língua inglesa, dificultando o seu uso por usuários que apenas conhecem outras línguas, como o Português (tabelas 1 e 2). Nos repositórios brasileiros, o número de OAs disponíveis ainda é pequeno e eles abordam poucas áreas do conhecimento. Por exemplo, em 2009, a Rede Interativa Virtual de Educação (RIVED) oferecia somente 190 OAs, sendo todos relativos ao ensino de ciências e matemática.

TABELA 1

Resultados de pesquisas por OAs da área de computação no repositório MERLOT ${ }^{22}$

\begin{tabular}{l|l|l}
\hline Palavras-chave & $\begin{array}{l}\text { Num. OAs } \\
\text { encontrados }\end{array}$ & Língua dos OAs \\
\hline Stack & 22 & $\begin{array}{l}21 \text { em inglês } \\
1 \text { em espanhol }\end{array}$ \\
\hline Pilha & 0 & --- \\
\hline Criptografia & 0 & --- \\
\hline Árvore B & 0 & --- \\
\hline Pipeline & 10 & 10 em ingles \\
\hline
\end{tabular}

TABELA 2

Resultados de pesquisas por OAs da área de computação no repositório $N S D L^{23}$

\begin{tabular}{l|l|l}
\hline \multicolumn{1}{c|}{ Palavras-chave } & \multicolumn{1}{c}{$\begin{array}{c}\text { Num. OAs } \\
\text { encontrados }\end{array}$} & \multicolumn{1}{c}{ Lingua dos OAs } \\
\hline Stack & 5.560 & 30 em inglês \\
\hline Pilha & 42 & $\begin{array}{l}6 \text { em inglês e } \\
24 \text { em português }\end{array}$ \\
\hline Criptografia & 2 & 2 em espanhol \\
\hline Árvore B & 0 & --- \\
\hline Pipeline & 10.028 & 30 em inglês \\
\hline
\end{tabular}

De acordo com Coelho e outros (2008), máquinas de busca nem sempre retornam resultados satisfatórios para alunos e professores. Os melhores resultados são retornados pela Google. Mas, mesmo assim, na média, somente 5\% do material retornado foi considerado um OA relevante, ou seja, um OA que obtenha nota mínima 3 dentre cinco critérios da avaliação LORI (NESBIT; BELFER; LEACOCK, 2003): qualidade do conteúdo, adequação para o objetivo, motivação, feedback e reusabilidade. Além disso, máquinas de busca retornam um grande volume de informação, o que faz com que alunos e professores gastem tempo na

\footnotetext{
${ }^{22}$ FONTE - As autoras, sendo a mesma das demais que aparecem neste trabalho.

${ }^{23}$ Somente foram acessados os 30 primeiros links da lista de resultados (IPROSPECT, 2006).
} 
seleção de material que lhes seja relevante. A Tabela 3 mostra alguns dos resultados obtidos utilizando Google.

TABELA 3

Resultados de pesquisas por OAs da área de computação utilizando Google

\begin{tabular}{l|r|rr}
\hline Palavras-chave & Num. resultados & Num. OAs relevantes & 0 \\
\hline Pilha & 2.890 .000 & 2 \\
\hline $\begin{array}{l}\text { Estrutura de dados } \\
\text { pilha }\end{array}$ & 184.000 & 3 \\
\hline Criptografia & 909.000 & 0 \\
\hline Segurança Criptografia & 298.000 & 0 \\
\hline Quicksort & 357.000 & & 0 \\
\hline
\end{tabular}

TABELA 4

Resultados de pesquisas por OAs da área de computação utilizando Delicious

\begin{tabular}{l|r|r}
\hline Palavras-chave & Num. resultados & Num. OAs relevantes \\
\hline Pilha & 291 & 1 \\
\hline $\begin{array}{l}\text { Estrutura de dados } \\
\text { pilha }\end{array}$ & 4 & 2 \\
\hline Criptografia & 1.779 & 6 \\
\hline Segurança Criptografia & 1 & 0 \\
\hline Quicksort & 399 & 0 \\
\hline
\end{tabular}

Ferramentas da Web 2.0 também podem ser usadas para recuperar OAs. Xu et al. (2008) mostraram que o uso de folksonomia pode gerar resultados melhores do que os obtidos por máquinas de busca baseadas na Web tradicional. Coelho e outros (2008) comprovaram essa afirmativa em um experimento. Segundo esses autores, $12 \%$ dos resultados das ferramentas da Web 2.0 eram links para OAs relevantes, o que é mais do que o dobro de resultados corretos $(5 \%)$ obtidos com pesquisas baseadas no uso de máquinas de busca tradicionais. A Tabela 4 mostra os resultados obtidos pelo uso do Delicious, que é uma das ferramentas mais conhecidas de bookmarking social (HAMMOND et al., 2005).

\section{VITAE}

VITAE é um acrônimo de Virtual and Interesting Teaching and learning material for Always and Ever, uma ferramenta projetada para auxiliar no compartilhamento de OAs. Ela está disponível em <http://bookmark.aspectos.org $>$.

VITAE segue o modelo de ferramenta de bookmarking social, porque foi o tipo de ferramenta da Web 2.0 que retornou melhores resultados em estudos de caso anteriores (COELHO et al., 2008). Bookmarkings sociais se destacam por permitirem a seus usuários 
armazenar links selecionados, compartilhá-los e organizá-los, pelo uso de tags. Além disso, são ferramentas simples e intuitivas, o que facilita o seu uso.

O desenvolvimento do VITAE considerou um conjunto de requisitos que foram definidos após análise das seguintes ferramentas da Web 2.0: Delicious ${ }^{24}$, Connotea $^{25}$, Gennio $^{26}$ e Citeulike $^{27}$. Essas ferramentas sociais foram selecionadas por sua popularidade, segundo o Alexa Traffic Rank, por serem muito citadas (HOTHO, 2006) e por sua usabilidade.

Os estudos realizados permitiram observar que as ferramentas de bookmarking social, apesar de possuírem características da Web 2.0, não ofereciam, no momento da pesquisa, recursos que permitam aos usuários avaliar o conteúdo das páginas para as quais os links apontam.

A definição dos requisitos também se baseou nas recomendações de Vuorikari (2007). Os requisitos são:

- Armazenamento de links para OAs com uso de tags para indexar os links.

- Pesquisa por links para OAs, usando tags como filtro de pesquisa.

- Avaliação de links para OAs realizada por usuários.

- Utilização da avaliação de links para OAs no ranqueamento dos resultados de uma pesquisa.

- Criação de contas para que os usuários possam postar links para OAs.

- Controle de acesso para assegurar a privacidade de usuários.

- Suporte para edição de tags.

O objetivo principal do VITAE é facilitar a recuperação de OAs. Para isso, a sua interface gráfica simplifica a experiência do usuário, por obedecer às seguintes recomendações de usabilidade (NIELSEN; LORANGER, 2006):

\footnotetext{
${ }^{24}$ Disponível em: 〈www.delicious.com>. Acesso em: 21 set. 2010.

${ }^{25}$ Disponível em: $\langle$ www.connotea.org $>$. Acesso em: 21 set. 2010.

${ }^{26}$ Disponível em: 〈gennio.com>. Acesso em: 21 set. 2010.

${ }^{27}$ Disponível em: 〈www.citeulike.org〉. Acesso em: 21 set. 2010.
} 
- Layout simples - a simplicidade ajuda o usuário a encontrar o que deseja, por facilitar a navegação. Elementos gráficos mal distribuídos ou animações exageradas retêm a atenção do usuário e, por isso, tornam a navegação difícil.

- Destaque de conteúdo - o uso de cores fortes e contrastes realçam o conteúdo. O objetivo é obter a atenção do usuário para o conteúdo e não para a página.

- Interface limpa - o uso de espaço em branco é um excelente recurso para organizar bem o conteúdo, por possibilitar uma separação bem definida dos tópicos, facilitando a navegação.

- Textos grandes - o texto que merece maior destaque deve utilizar o tamanho da fonte maior que a normal. Essa característica também facilita o uso do aplicativo pelos deficientes visuais.

No VITAE, os usuários podem realizar as seguintes atividades: pesquisar, salvar, associar tags e avaliar OAs associados a links.

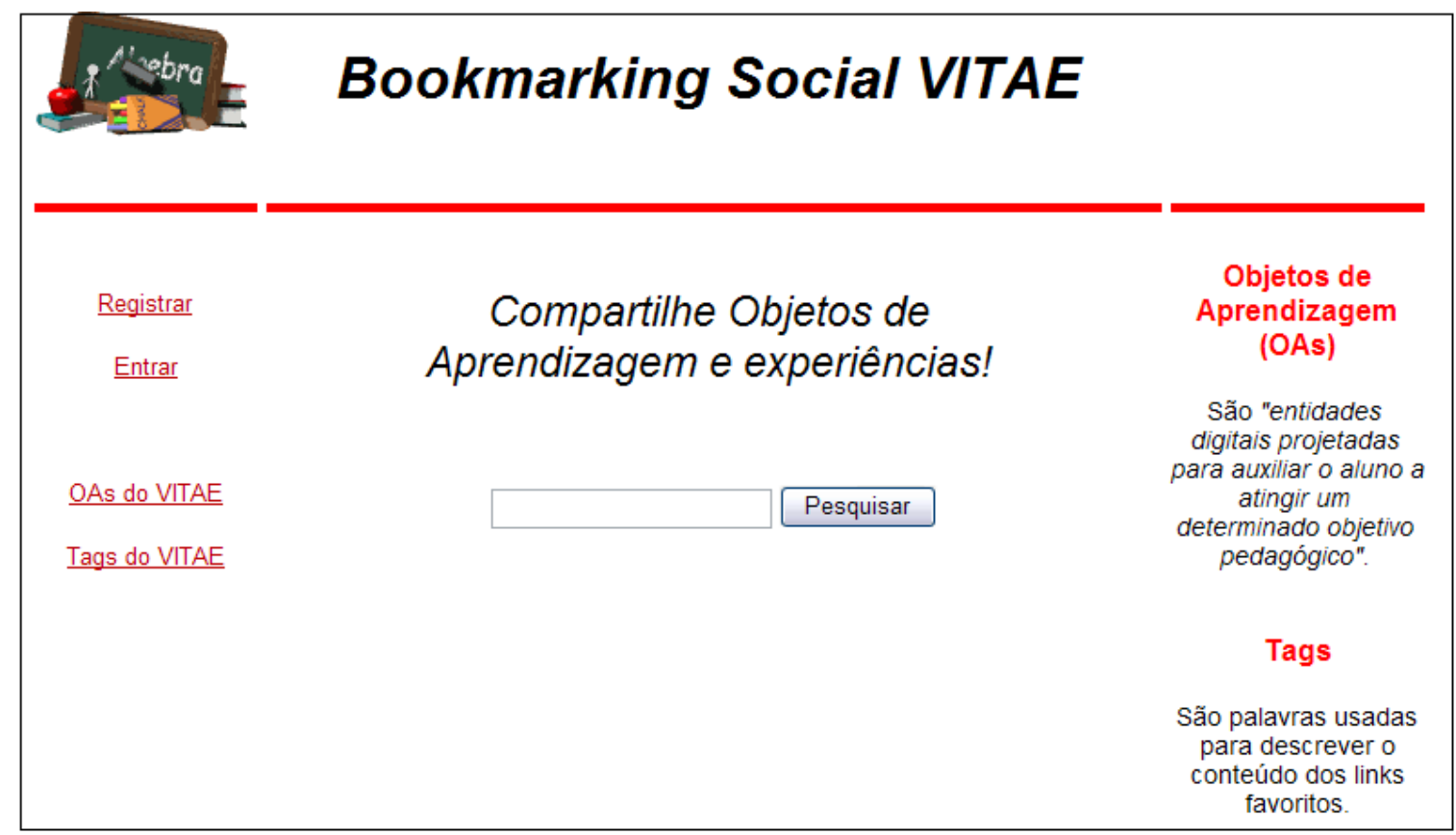

FIGURA 1 - Tela inicial do VITAE ${ }^{28}$

A pesquisa por OAs é baseada no uso de uma ou mais tags. Na tela inicial apresentada na Figura 1, o usuário digita uma ou mais tags no campo de texto e clica no botão "Pesquisar". VITAE pesquisa por tags relacionadas, armazenadas no banco de dados. Os links 
resultantes são mostrados ao usuário, ordenados pela nota média obtida nas avaliações realizadas pelos usuários (Figura 2).

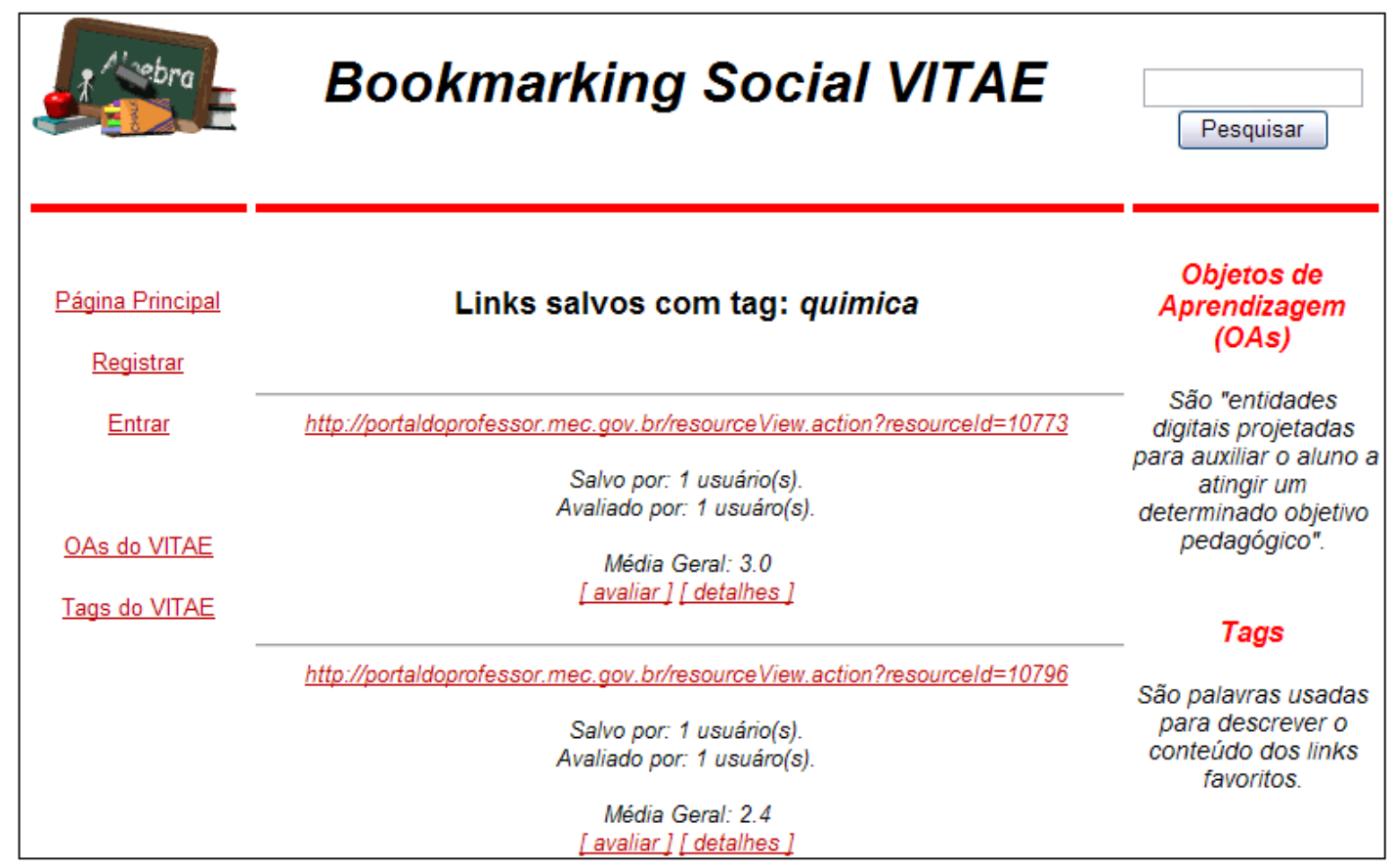

FIGURA 2 - Resultados de uma pesquisa baseada na tag "química"

Para salvar um link para OA, o usuário deve preencher o formulário mostrado na Figura 3.

${ }^{28}$ FONTE - As autoras, sendo a mesma das que aparecem neste trabalho. 


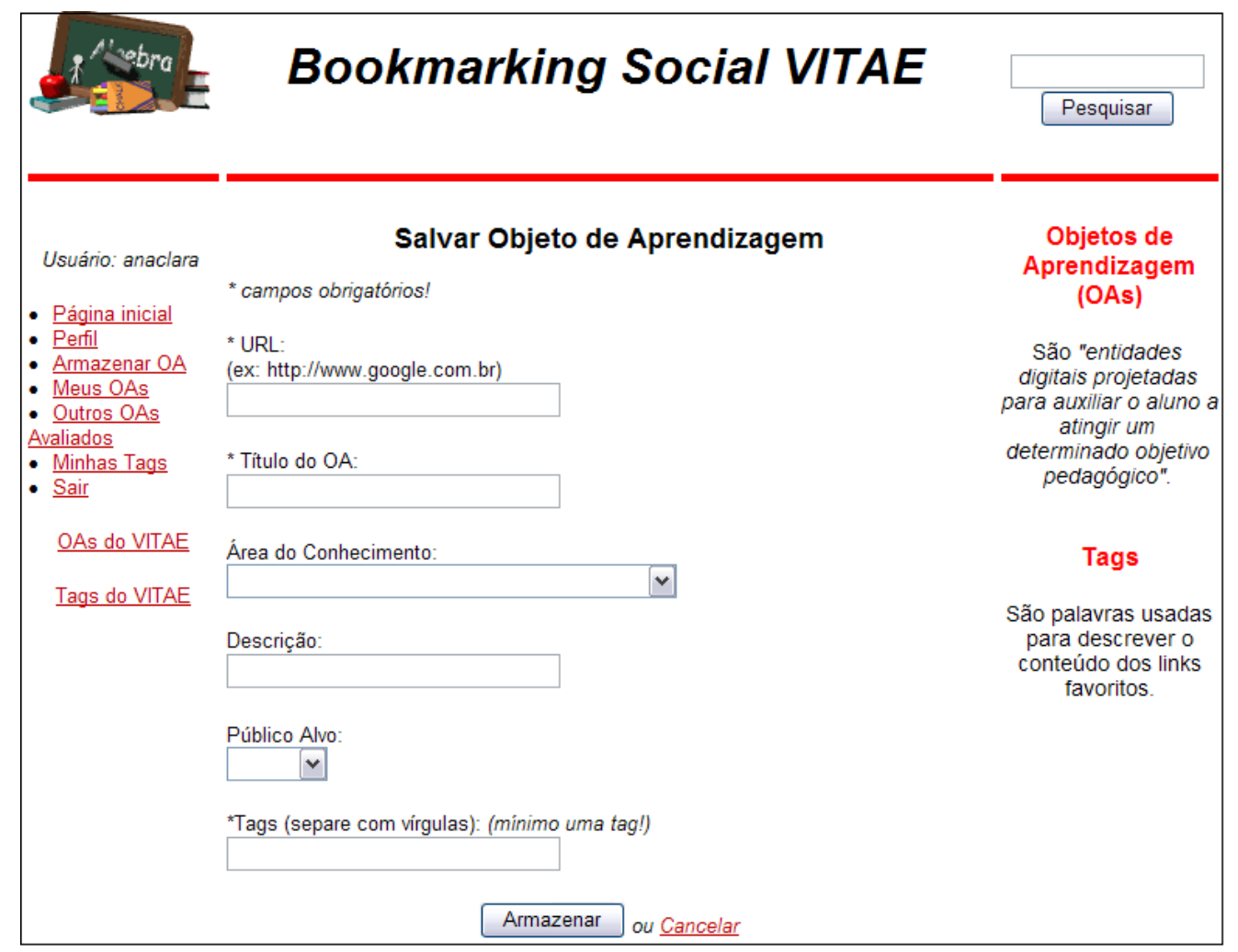

FIGURA 3 - Inserção de links para OAs

Usuários também podem avaliar OAs apontados por links armazenados no VITAE. Baseados no Learning Object Review Instrument (LORI) (NESBIT; BELFER; LEACOCK, 2003), os cinco aspectos que podem ser avaliados são: qualidade de conteúdo, objetivo de aprendizagem, feedback, capacidade de motivação e reusabilidade (Figura 4). Cada um desses aspectos pode ser avaliado com notas que variam de 1 a 5 . Após finalizar o processo de avaliação, o sistema mostra a média geral do OA, considerando todas as avaliações atribuídas a ele (Figura 5). O sistema só armazena o resultado de uma avaliação quando todos os critérios tiverem sido avaliados. 


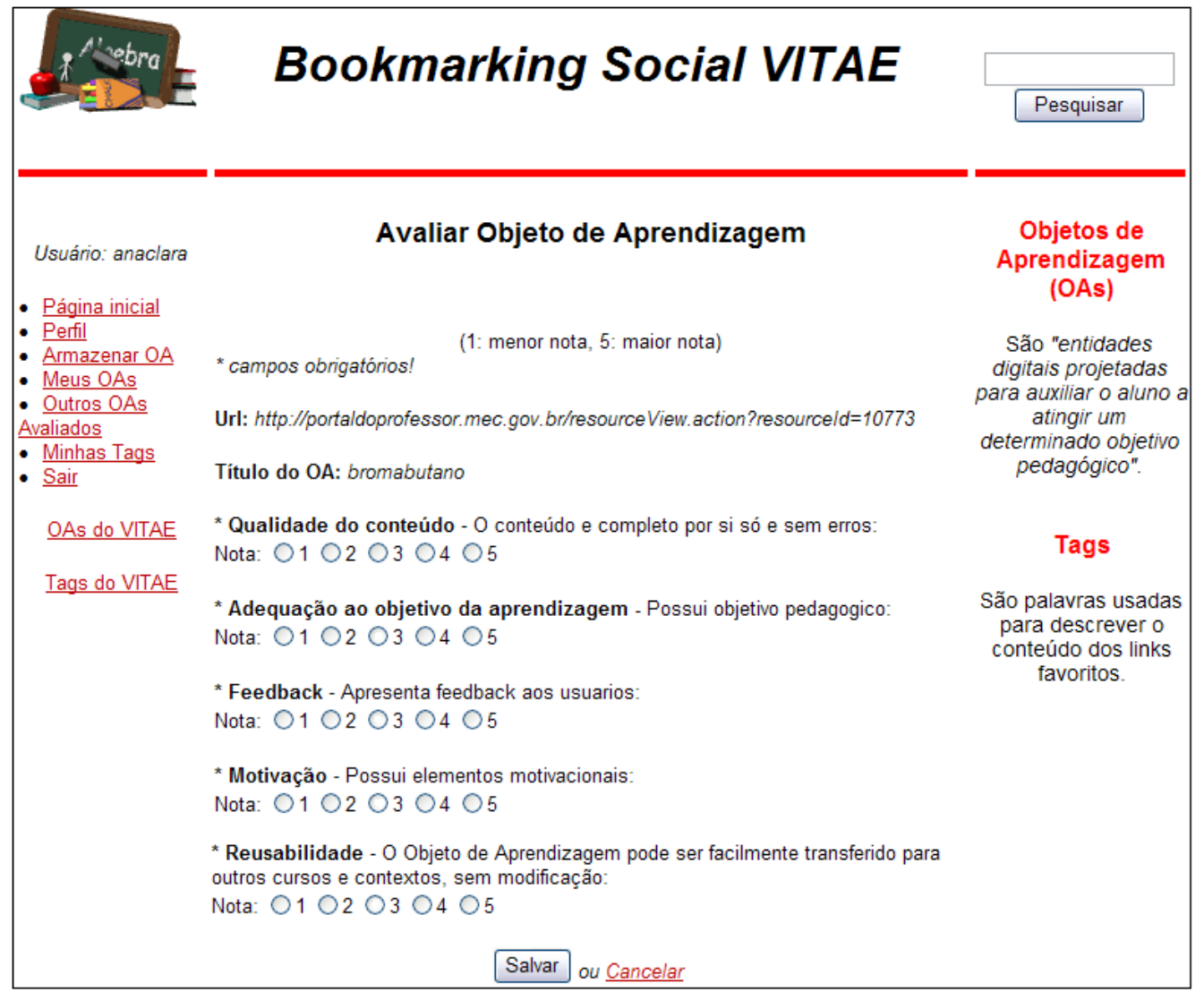

FIGURA 4 - Avaliação de OA

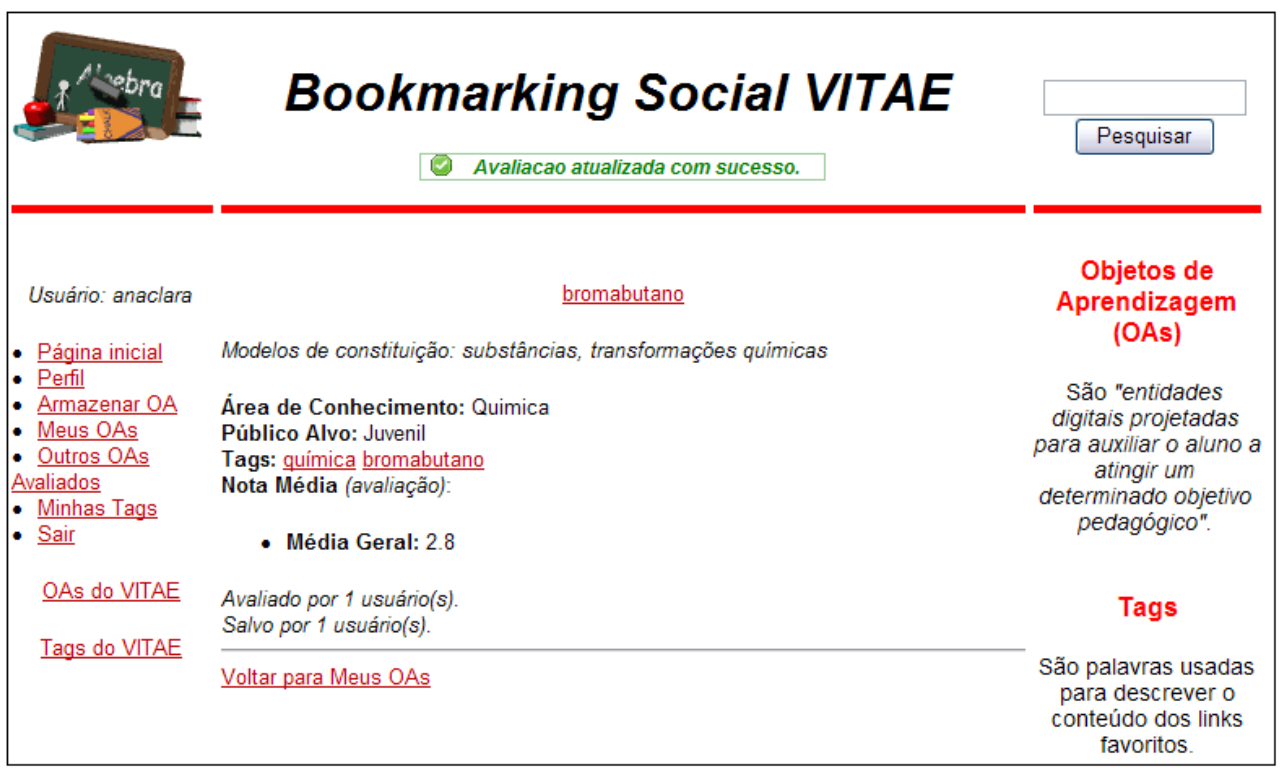

FIGURA 5 - Resumo de avaliação de OA 


\section{AVALIAÇÃO DO VITAE}

A avaliação da ferramenta se baseou na comparação com outras, em critérios de usabilidade $^{29}$ e na análise de seu uso.

Para que o processo de avaliação pudesse ser realizado, seu banco de dados foi preenchido com links de OAs encontrados em repositórios e sites de professores que disponibilizam material didático. Esses links foram extraídos da Web por um software escrito em PHP (Hypertext Preprocessor), desenvolvido especificamente para a realização dessa tarefa. Essa estratégia foi utilizada apenas no momento da publicação da ferramenta, para que os usuários não se sentissem desmotivados em realizar buscas que ainda não apresentassem resultados. Após esse primeiro momento, os usuários se tornam responsáveis por manter todas as atividades no bookmarking social: a pesquisa, o armazenamento e a avaliação dos OAs apontados pelos links.

VITAE foi comparado com repositórios e ferramentas da Web 2.0. Os repositórios considerados na comparação foram os seguintes: RIVED ${ }^{30}, \operatorname{LabVirt}^{31}, E d u k b r^{32}, O E 3^{33}$, MERLOT ${ }^{34}, N S D L^{35}$ e Ariadne ${ }^{36}$. E as aplicações 2.0 foram: Digg ${ }^{37}$, StumbleUpon ${ }^{38}$, Delicious $^{39}$ e TeacherTube ${ }^{40}$, escolhidas devido à sua popularidade.

Na comparação, o VITAE se destacou por suas características inovadoras, como:

- a possibilidade de avaliação de objetos de aprendizagem, sem restrições. A avaliação é uma característica que contribui para a melhoria dos OAs, pois permite que desenvolvedores tenham acesso aos aspectos que devem ser melhorados. Além disso, a avaliação facilita a seleção de OAs pelos usuários, pois as notas atribuídas aos OAs são utilizadas no ranqueamento de resultados;

\footnotetext{
${ }^{29}$ Usabilidade é "um atributo de qualidade relacionado à facilidade do uso de algo. Mais especificamente, referese à rapidez com que os usuários podem aprender a usar alguma coisa, a eficiência deles ao usá-la, o quanto lembram daquilo, seu grau de compreensão a erros e o quanto gostam de utilizá-la" (NIELSEN; LORANGER, 2006, p. xvi).

${ }^{30}$ Disponível em: <rived.mec.gov.br〉.

${ }^{31}$ Disponível em: 〈www.labvirt.fe.usp.br>. Acesso em: 21 set. 2010.

${ }^{32}$ Disponível em: <www.edukbr.com.br>. Acesso em: 21 set. 2010.

${ }^{33}$ Disponível em: $\langle\underline{w w w . c e s e c . u f p r . b r / e t o o l s / o e 3}\rangle$. Acesso em: 21 set. 2010.

${ }^{34}$ Disponível em: 〈www.merlot.org〉. Acesso em: 21 set. 2010.

${ }^{35}$ Disponível em: $\langle$ nsdl.org $>$. Acesso em: 21 set. 2010.

${ }^{36}$ Disponível em: 〈www.ariadne-eu.org >. Acesso em: 21 set. 2010.

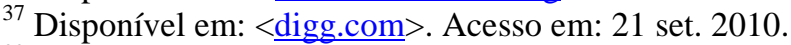

${ }^{38}$ Disponível em: 〈www.stumbleupon.com>. Acesso em: 21 set. 2010.

${ }^{39}$ Disponível em: $\langle$ www.delicious.com>. Acesso em: 21 set. 2010.

${ }^{40}$ Disponível em: 〈www1.teachertube.com>. Acesso em: 21 set. 2010.
} 
- $\quad$ suporte para exclusão de links para OAs - esse recurso oferece ao usuário a liberdade para excluir links para OAs, quando estes são irrelevantes, incorretos ou desatualizados.

Além dessas características, VITAE permite que os usuários armazenem links para OAs sem a necessidade de aprovação de terceiros nem de conhecimento e preenchimento de esquemas de metadados exigidos por repositórios. No VITAE, o processo de armazenamento é muito simples.

Para avaliação de usabilidade, foi elaborado um checklist, contendo diretrizes sugeridas por Nielsen e Loranger (2006). Esse checklist incluiu 20 aspectos que avaliavam o uso de cores e animações, o uso do botão "voltar", o uso de janelas pop-up, o sistema de pesquisa, a concisão, a consistência e a correção do conteúdo, a rolagem de tela, a portabilidade do site.

VITAE foi avaliado por 29 alunos e professores de duas instituições de ensino: Instituto Federal de Minas Gerais - campus São João Evangelista (IFMG-SJE) e Pontifícia Universidade Católica de Minas Gerais (PUC MINAS), sendo que a maior parte dos avaliadores eram professores. Alguns destes tinham conhecimento sobre OAs e, inclusive, os desenvolviam para sua prática profissional. No entanto, não estavam habituados a acessar e a recuperar OAs através de repositórios, mesmo conhecendo o RIVED.

Para essa avaliação, foi elaborado um conjunto de cinco afirmativas que deveriam ser confirmadas pelos usuários. As afirmativas e os resultados obtidos estão apresentados na Tabela 5. Os resultados mostram que $86 \%$ dos usuários concordam com a afirmativa de que o VITAE é fácil de usar. A afirmativa que recebeu o menor número de concordâncias $-69 \%$ dos usuários - foi a terceira, o que indica a necessidade de melhorar a qualidade das orientações apresentadas no site. Como todas as demais afirmativas obtiveram um mínimo de $80 \%$ de concordância, pode-se concluir que os usuários avaliaram positivamente o uso do VITAE. 
TABELA 5

Resultados da avaliação do VITAE pelos usuários

\begin{tabular}{|c|c|c|c|c|c|}
\hline \multirow[t]{2}{*}{ Afirmativa } & \multicolumn{5}{|c|}{ Opinião dos usuários } \\
\hline & $\begin{array}{l}\text { Concordo } \\
\text { fortemente }\end{array}$ & Concordo & Indeciso & Discordo & $\begin{array}{l}\text { Discordo } \\
\text { fortemente }\end{array}$ \\
\hline O VITAE é fácil de usar. & 14 & 11 & 2 & 2 & 0 \\
\hline $\begin{array}{l}\text { Os recursos de navegação } \\
\text { (menus, ícones e botões) } \\
\text { estão todos claros e } \\
\text { fáceis de achar. }\end{array}$ & 12 & 14 & 1 & 2 & 0 \\
\hline $\begin{array}{l}\text { As informações são } \\
\text { objetivas. }\end{array}$ & 10 & 10 & 6 & 2 & 1 \\
\hline $\begin{array}{l}\text { As telas possuem } \\
\text { informações de fácil } \\
\text { visualização. }\end{array}$ & 12 & 11 & 1 & 5 & 0 \\
\hline $\begin{array}{l}\text { As telas facilitam a } \\
\text { leitura e a identificação } \\
\text { dos campos de entrada de } \\
\text { dados e seus formatos (ex: } \\
\text { urls, e-mail). }\end{array}$ & 12 & 13 & 1 & 3 & 0 \\
\hline
\end{tabular}

\section{CONCLUSÕES E TRABALHOS FUTUROS}

Objetos de aprendizagem podem motivar estudantes, com interações e interfaces gráficas. Entretanto, a falta de tempo e de conhecimentos técnicos representa dificuldades para os professores desenvolverem materiais educacionais com conteúdo e características multimídia ricas, que atendam aos objetivos de um OA. Uma alternativa é reutilizar OAs disponíveis, que sejam de boa qualidade. Para isso, é necessário usar mecanismos que facilitem a pesquisa e a recuperação de OAs. Mecanismos existentes não são eficientes no resultado de pesquisas por OAs (COELHO et al., 2008). Repositórios de OAs, conforme discutido na Seção 3, nem sempre são conhecidos e, quando o são, são restritos nas áreas de conhecimento que cobrem.

Conforme discutido na Seção 3, máquinas de busca podem auxiliar na pesquisa por OAs disponíveis na Web; entretanto, raramente retornam links para objetos de aprendizagem de boa qualidade (NESBIT; BELFER; LEACOCK, 2003) ou, com frequência, colocam os OAs mal ranqueados na lista de resultados. Além disso, nem sempre as máquinas de busca encontram OAs disponíveis em repositórios.

Essas dificuldades motivaram a pesquisa por outros mecanismos de recuperação de OAs. Conforme estudos recentes, ferramentas Web 2.0 produzem melhores resultados do que máquinas de busca. Esse contexto motivou a proposta de uma ferramenta de recuperação de OAs, baseada na Web 2.0. Em especial, as ferramentas de bookmarking social possuem uma característica que atende a essa proposta: a possibilidade de armazenar e compartilhar links 
para OAs. Nas ferramentas já existentes de bookmarking social, foi observado que usuários podem adicionar links sem restrições, o que não ocorre em repositórios. Entretanto, não foi encontrada ferramenta que permitisse avaliar esses links, ou, mais especificamente, os OAs associados a cada link.

Essas observações permitiram definir um conjunto de requisitos e funcionalidades que uma ferramenta Web 2.0 deve ter, para apoiar a recuperação de OAs. Para atender ao requisito de avaliação de OAs, este artigo propôs uma ferramenta de bookmarking social que incorpora a avaliação de OAs. Esses requisitos foram considerados na implementação do protótipo denominado VITAE.

Quando comparado a outros repositórios e ferramentas de busca, VITAE apresenta algumas características inovadoras, como a possibilidade de remover ou avaliar OAs. A avaliação de OAs contribui para melhorar os resultados da pesquisa, porque as notas atribuídas a cada OA são utilizadas como critério de ranqueamento.

VITAE foi avaliado por um grupo de usuários, que o considerou fácil de usar, fácil de navegar, com informação objetiva e com campos de fácil visualização. Os resultados obtidos demonstram a aplicabilidade da proposta.

Muitos estudos podem ser desenvolvidos a partir dos resultados apresentados neste trabalho. Uma possibilidade seria desenvolver uma nova versão, melhorada, do VITAE, que incorpore características como: desenvolvimento de um critério de ranqueamento personalizado ao perfil de cada usuário, criação de grupos de usuários para discussão, uso de RSS para manter usuários atualizados.

Além desses recursos, VITAE também necessita de melhores técnicas para indexação e recuperação de OAs.

Do ponto de vista pedagógico, cabe avaliar o impacto do uso do VITAE no ambiente acadêmico, como ferramenta de suporte à educação.

Assim, sob o aspecto multidisciplinar, espera-se que o VITAE sirva como base para várias pesquisas não somente na área de computação, mas também na área de educação, contribuindo para a melhoria contínua do processo de ensino e aprendizagem. 


\section{REFERÊNCIAS}

ADVANCED DISTRIBUTED LEARNING. SCORM. 2004. Disponível em:

<http://www.adlnet.gov/Technologies/scorm/default.aspx >. Acesso em: 21 set. 2010.

ANDERSON, Paul. What is Web 2.0? Ideas, technologies and implications for education.

Journal of Information Science, fev. 2007. Disponível em:

<http://www.jisc.ac.uk/media/documents/techwatch/tsw0701b.pdf $>$. Acesso em: 21 set. 2010.

COELHO, Geovália Oliveira. et al. Enhancing access to learning objects for lifelong learning. In: IFIP WORLD CONFERENCE ON COMPUTERS IN EDUCATION (WCCE 2009), 9., 2009, Bento Gonçalves. Proceedings... Bento Gonçalves: WCCE, 2009.

COELHO, Geovália Oliveira. et al. Estudo e Proposta de Recuperação de Objetos de Aprendizagem Usando a WEB 2.0. In: BRAZILIAN WORKSHOP ON SEMANTIC WEB AND EDUCATION (SBIE 2008), 2, 2008, Fortaleza. Proceedings... Fortaleza: SBC, 2008.

FABRE, Marie-Christine Julie Mascarenhas; TAROUCO, Liane Margarida Rockenbach; TAMUSIUNAS, Fabrício Raupp. Reusabilidade de objetos educacionais. RENOTE Revista Novas Tecnologias na Educação, Porto Alegre, v. 1, n. 1, 2003.

GONZALEZ-BARBONE, Victor; ANIDO-RIFON, Luis. Creating the first SCORM object. Computers \& Education, v. 51, n. 4, p. 1634-1647, 2008.

HAMMOND, Tony. et al. Social bookmarking tools. D-Lib Magazine, v. 11, n. 4, 2005.

HOTHO, Andreas. et al. Information retrieval in folksonomies: search and ranking. In: Annual European Semantic Web Conference, 3., 2006, Budva, Montenegro. Proceedings... Budva: Springer, p. 411-426, 2006.

IPROSPECT. Search engine user behavior study. 2006. Disponível em: $<$ http://www.iprospect.com/premiumPDFs/WhitePaper_2006_SearchEngineUserBehavior.pd f>. Acesso em: 20 set 2010 .

LEARNING TECHNOLOGY STANDARDS COMMITTEE. Draft Standard for Learning Object Metadata. IEEE Standard 1484.12.1. 2002. Disponível em:

<http://ltsc.ieee.org/wg12/files/LOM_1484_12_1_v1_Final_Draft.pdf > . Acesso em: 20 set 2010.

NESBIT, John; BELFER, Karen; LEACOCK, Tracey. Learning object review instrument (lori). E-Learning Research and Assessment Network, 2003. Disponível em: <http://www.elera.net/eLera/Home/Articles/LORI\%201.5.pdf> . Acesso em: 20 set. 2010.

NIELSEN, Jakob; LORANGER, Hoa. Prioritizing web usability. California: New Riders, 2006. 
O'REILLY, Tim. What is Web 2.0 - design patterns and business models for the next generation software. 2005. Disponível em: <http://oreilly.com/web2/archive/what-is-web20.html>. Acesso em: 20 set. 2010.

\section{SAINT IGNATIUS HIGH SCHOOL. The World's Most Widely Spoken Languages.}

Disponível em: <http://www2.ignatius.edu/faculty/turner/languages.htm Acesso em: 20 mar. 2011.

VUORIKARI, Riina. Can social information retrieval enhance the discovery and reuse of digital educational content? In: ACM CONFERENCE ON RECOMMENDER SYSTEMS, 2007, Minneapolis. Proceedings... New York: ACM, 2007. p. 207-210.

XU, Shengliang. et al. Exploring folksonomy for personalized search. In: ANNUAL INTERNATIONAL ACM SIGIR CONFERENCE ON RESEARCH AND DEVELOPMENT IN INFORMATION RETRIEVAL, 31, 2008, Singapore. Proceedings... New York: ACM, 2008. p. 155-162.

\section{Como citar este artigo:}

COELHO, Geovália Oliveira; ISHITANI, Lucila; NELSON, Maria Augusta Vieira. VITAE: recuperação de objetos de aprendizagem baseada na web 2.0. ETD - Educ. temat. digit., Campinas, SP, v.14, n.2, p.238-257, jul./dez. 2012. ISSN 1676-2592. 